\title{
Spectroscopic study of charmed baryons at J-PARC
}

\author{
Hiroyuki Noumi ${ }^{* \dagger}$ \\ RCNP, Osaka-U \\ E-mail: noumi@rcnp.osaka-u.ac.jp
}

Charmed baryons with a charm quark provide a unique opportunity to investigate quark dynamics, particularly diquark correlations, in baryons. Because a charm quark is heavy and spin-dependent interactions with the charm quark is weaker, charmed baryons are characterized by the motions of the other two light-quarks. We demonstrate that the nature of light- $q q$ diquark motions in charmed baryons appears in the level structure, production rates, and decay branching ratios. We propose a spectroscopic study of charmed baryons via the $p\left(\pi^{-}, D^{*-}\right)$ reaction at J-PARC. Systematic measurements of the excitation energy spectrum and decays of charmed baryons can be carried out by means of the missing mass technique.

XV International Conference on Hadron Spectroscopy

4-8/11/2013

Nara, Japan

* Speaker.

${ }^{\dagger}$ This article is based on works of the P50 collaboration and discussions with A. Hosaka and his co-workers. 


\section{Introduction}

It is a fundamental question in hadron physics how hadrons form. The principle to describe the dynamics of quarks is known as the Quantum Chromodynamics (QCD). However, it is still difficult to reproduce hadrons, especially excited hadrons, from bare quarks and gluons by solving the equation of QCD due to its non-perturbative nature in low energy. In the constituent quark model (CQM), quarks are treated as an effective degree of freedom in hadrons. In the CQM, light $u, d$, and $s$ quarks are renormalized due to the strong interaction, as "dressed" quarks, while heavy $c$ and $b$ quarks act as constituent quarks. The CQM rather well describes the properties of hadrons, such as classification based on spin/flavor symmetry, mass relations, magnetic moments of ground state baryons, and so on. However, the situation is different in excited states. There are many undiscovered states predicted by CQM, which is known as the so-called missing resonance problem. The mass order of resonances, i.e. the $\mathrm{N}^{*}(1440) 1 / 2^{+}$, and/or $\Lambda(1405) 1 / 2^{-}$state, is not clearly explained. Recently, unexpected, narrow states in excited hadrons, such as $\Theta^{+}, \mathrm{X}, \mathrm{Y}, \mathrm{Z}$, and $\mathrm{Z}_{b}$ [1, 2], have been reported. These states are not yet understood very well.

Above-mentioned situation indicates rich structure of hadrons. We need to investigate the interaction between quarks in hadrons further. In particular, roles of quark-quark correlation in a baryon, namely diquark correlation, has yet to be understood, although existence or even evidence of diquarks in many hadronic processes has been discussed for a long time [3 4 5 6]. In light baryons, where flavor SU(3) symmetry seems to work rather well, 3 diquark pairs are expected to be correlated each other at an equal weight. Extraction of diquark correlation may not be easy. When a quark in a light baryon is replaced by a heavy quark, one expects that motions of light quarks are separated from that of a heavy quark. Since magnitude of the spin-spin interaction between quarks is proportional to the inverse of the quark mass, a correlation between light quarks may become much stronger. In this respect, diquark correlation is expected to be singled out in a baryon with a heavy quark. Lattice QCD calculations demonstrate strong spatial correlations between two light quarks with a spin-singlet, color anti-triplet configuration in a baryon with introducing a static quark [7 8]. Therefore, baryons with a charm quark provide unique opportunities to study diquark correlation. The nature of the diquark correlation is expected to appear in level structure, production rates, and decay branching ratios, as described in the following sections. We therefore propose a spectroscopic study of charmed baryons via the $p\left(\pi^{-}, D^{*-}\right)$ reaction, where systematic measurements of the level structure, production rates, and branching ratios of charmed baryons in a wide mass range can be carried out by means of a missing mass technique [9].

\section{Level Structure}

If a quark in a light baryon is replaced by a heavier quark, orbital excited states split into a relative motion in the light quarks ( $\rho$ mode) and a collective motion of them to the heavy quark ( $\lambda$ mode), as illustrated in Fig. 1 This kind of level splitting at orbital excited states occurs when a confinement potential is given. A ratio of $\rho$-mode excitation energy to $\lambda$-mode one is given as $\omega_{\rho} / \omega_{\lambda}=\sqrt{3 m_{Q} /\left(2 m_{q}+m_{Q}\right)}$ in the case of a harmonic oscillator potential, where $m_{q}$ and $m_{Q}$ correspond to constituent quark masses of light and heavy quarks, respectively. This is the socalled isotope shift. The levels further split due to the spin-dependent interactions. In particular, 


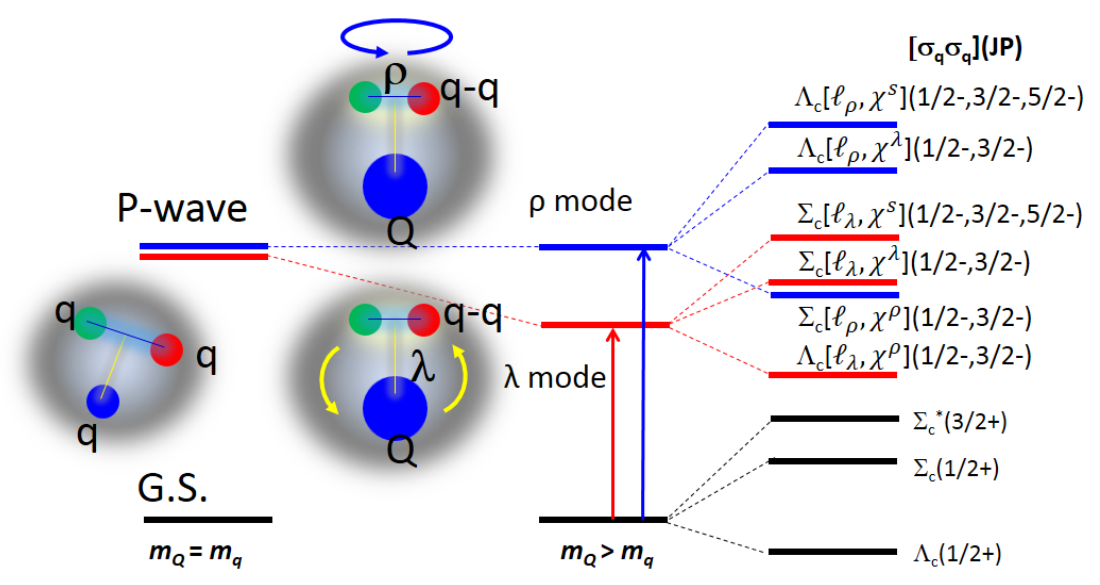

Figure 1: Schematic picture of quark correlations in a baryon. In a baryon with light quarks, the correlations are equal weight and the orbital excitation levels are degenerated, as illustrated in the left hand side. In the case that two light quarks make a collective system by introducing a heavy quark in a baryon, the orbitally excited states split into two: a relative orbital motion between two light quarks and a collective motion of the light quarks relative to the heavy quark. The levels further split due to the spin-dependent interactions.

the spin-spin interaction, denoted as $V_{s s} \sim \sum_{i, j} \sigma_{i} \cdot \sigma_{j} / m_{i} m_{j}$, characterizes the level structure. Antisymmetrizing a fermion system, configurations of orbital angular momentum $\left(\ell_{\rho}\right.$ or $\left.\ell_{\lambda}\right)$, spin $\left(\chi^{\rho}, \chi^{\lambda}\right.$, or $\left.\chi^{s}\right)$, isospin $(\Lambda$, or $\Sigma)$, and colors (antisymmetric) are determined. Here, $\ell_{\rho(\lambda)}$ and $\chi^{\rho(\lambda)}$ represent antisymmetric (symmetric) orbital and spin configurations of light quarks, respectively. A symmetric configuration for 3 quark spins is represented by $\chi^{s}$. Schematic level structure of charmed baryons are illustrated in the right-hand side of Fig. 1. The level structure reflects a motion of a light-quark pair.

\section{Production rate}

We consider the t-channel $D^{*}$ exchange model at a forward scattering angle in order to estimate the production rates for the excited charmed baryons, as shown in Fig. 2. (a). Here, a $u$ quark in the proton is converted to a $c$ quark to form an excited charmed baryon. Let us consider that a $u d$ diquark in a baryon acts as a spectator in the reaction. Then, the production rate is expressed as:

$$
R \sim \gamma C|K \dot{I}|^{2} p_{B}
$$

where $\gamma$ stands for a kind of spectroscopic factor to pick up good or bad diquark configuration in the target proton. A naive quark model gives the $\gamma$ of $1 / 2(1 / 6)$ for the good (bad) diquark configuration. $C$ is a spin dependent coefficient, which is the products of the Clebush-Gordan coefficients based on the quark-diquark spin configurations in the initial and final baryon states. $K$ is a kinematic factor expressed as:

$$
K \sim k_{D^{*}}^{0} k_{\pi}\left(\left|\mathbf{p}_{\mathbf{B}}\right| / 2 m_{B}-1\right) /\left(q^{2}-m_{D^{*}}^{2}\right),
$$

Then, $I$ is the overlap integral of the initial and final states.

$$
I \sim \sqrt{2} \int d \mathbf{r}^{3}\left[\varphi_{f}^{*}(\mathbf{r}) e^{\mathbf{q}_{\text {effr }}}\left[\varphi_{i}(\mathbf{r})\right],\right.
$$


(a)

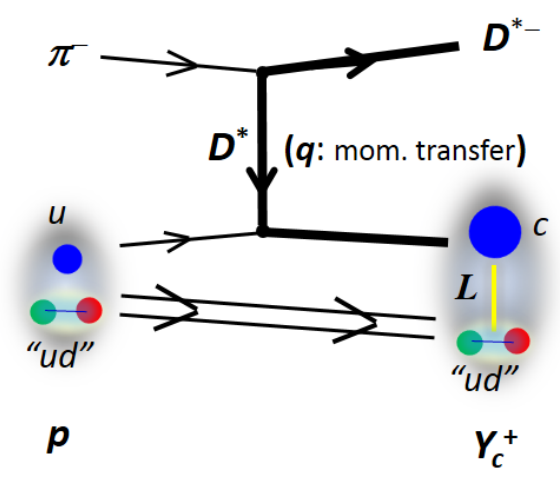

(b)

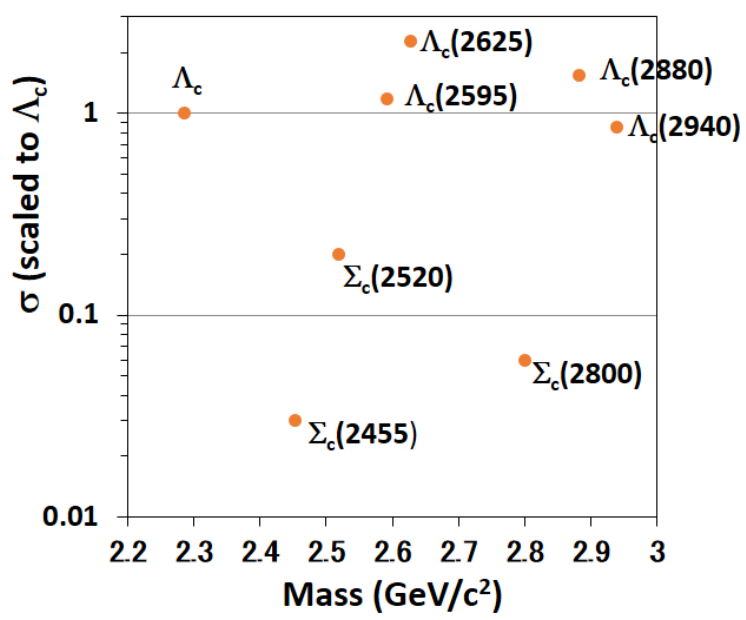

Figure 2: (a) Diagram of the $p\left(\pi^{-}, D^{*-}\right) \Lambda_{c}^{+}$reaction. The t-channel $D^{*}$ exchange reaction is considered at a forward scattering angle. (b) Production rate relative to the ground state estimated in the $p\left(\pi^{-}, D^{*-}\right) Y_{c}^{+}$ reaction at $p_{\pi}=20 \mathrm{GeV} / c$. 111

$$
\mathbf{q}_{\mathbf{e f f}}=\mathbf{p}_{\mathbf{p}} \times \frac{m_{d}}{M_{p}}-\mathbf{p}_{\mathbf{Y}_{\mathbf{c}}} \times \frac{m_{d}}{M_{Y_{c}}},
$$

where $\varphi_{i}(\mathbf{r})$ and $\varphi_{f}(\mathbf{r})$ are the wave functions of the initial and final quark states, respectively. The effective momentum transfer $\mathbf{q}_{\text {eff }}$ represents a recoil effect, where $m_{d}$ stands for the residual $u d$ diquark mass. Taking harmonic oscillator wave functions, $I$ can be expressed as,

$$
I \sim\left(q_{e f f} / A\right)^{L} e^{-q_{e f f}^{2} / 2 A^{2}},
$$

where $A$ is the average of the oscillator parameters of the initial and final wave functions. A typical value of $A$ can be taken as $0.4 \sim 0.45 \mathrm{GeV}$. The quantity $L$ is the orbital angular momentum of the excited baryon. This expression shows some interesting features for $I$. The relative strength of $I(L)$ for the excited state to $I(0)$ for the ground state is proportional to $\left(q_{e f f} / A\right)^{L}$ if we ignore the small difference of $q_{e f f} / A$. In the case of $q_{\text {eff }}$ greater than $A$, the relative rate increases as $L$ increases. One finds that $I$ is maximum at $q_{e f f}=\sqrt{2} A$. This can be called as the momentum matched condition. For larger $q_{e f f}$, the absolute value of $I$ decreases rapidly.

We summarize the production rates relative to the ground state estimated in the cases of the $p\left(\pi^{-}, D^{*-}\right) Y_{c}^{+}$reaction at $p_{\pi}=20 \mathrm{GeV} / c$ in Fig. 2-(b) [11]. The present model calculation suggests that the production rate is kept even at the higher $L$ states due to a large momentum transfer of greater than $1 \mathrm{GeV} / c$ in the reaction, although an absolute cross section is expected to be much smaller, as described in Section 5.1. One finds that the production rate strongly depends on spin/isospin structures of baryons. It should be noted that the reaction is expected to favor the $\lambda$ mode excitations. The production rates of excited states with different isospin and orbital angular momentum will provide the information on the spin and radial wave functions of charmed baryons.

\section{Decay}

We consider decays of excited charmed baryons. On one hand, a string between the light- $q q$ 
diquark and a charm quark will be expanded in an $\lambda$-mode excited state. If the excited energy is sufficiently high, a $q \bar{q}$ pair is created in a string. This may favor a decay to a heavy meson $(Q \bar{q})$ and a light baryon $(q q q)$ rather than a decay to a heavy baryon $(Q q q)$ and a light meson $(q \bar{q})$. On the other hand, the situation may be opposite in an $\rho$-mode excited state. Measurements of decay branching ratios provide information on the motion of the $q q$ diquark in a excited charmed baryon.

\section{Charmed Baryon Spectroscopy via the $p\left(\pi^{-}, D^{*-}\right)$ Reaction}

We propose a spectroscopic study of charmed baryons via the $\mathrm{p}\left(\pi^{-}, D^{*-}\right)$ reaction at the $\mathrm{J}$ PARC high-momentum beam line 9 . The high-momentum beam line is designed to deliver highintensity pions of $\sim 10^{7}$ every second at $20 \mathrm{GeV} / c$. A dispersive beam optics is realized so that a pion beam momentum can be measured with a resolution of $0.1 \%$ at a beam focal plane. A magnetic spectrometer is designed for scattered $D^{*-}$, which can covers a large acceptance of greater than $50 \%$. Charmed baryons with a wide mass range are expected to be observed in a missing mass spectrum with an energy resolution of $\sim 10 \mathrm{MeV}$, where the charmed baryons can be identified independent of their decay final states. Owing to the large acceptance of the spectrometer, we can detect decay particles emitted from observed charmed baryons in a wide angular range, and can measure decay branching ratios as well as spin-parity. More detailed description of the beam line and spectrometer can be found elsewhere [10].

\subsection{Cross section}

Estimation of the cross section of the $p\left(\pi^{-}, D^{*-}\right) \Lambda_{c}^{+}$reaction is an experimental issue, which affects an experimental design in order to achieve reasonable signal sensitivity. However, available data on the reaction are limited. An upper limit, $7 \mathrm{nb}(68 \% \mathrm{CL})$ at the incident pion momentum of $13 \mathrm{GeV} / \mathrm{c}$, reported at BNL [12], suggests a small cross section of charmed baryons at a level of nb.

We employ the Regge model to estimate the cross section as it well describes binary (twobody) reactions at high energy [13]. In the Regge theory, the differential cross section of a binary reaction shows a typical $s$-dependence in the limit $s \rightarrow \infty$ :

$$
\frac{d \sigma}{d t}=\frac{g_{1}^{2} g_{2}^{2}}{64 \pi\left|p_{1}\right|^{2} s} \Gamma^{2}(-\alpha(t))\left(\frac{s}{s_{0}}\right)^{2 \alpha(t)}
$$

where $\alpha(t)$ is the Regge trajectory [14] and the scale parameter $s_{0}$ stands for the square of the threshold energy in the reaction. The above formula of the cross section is applicable at the diffractive region of $|t| \ll s$, where the typical angular distribution decreases rapidly as $|t|$ increases. We ignore the $t$ dependence of the Gamma function as $\Gamma\left(-\alpha\left(t_{\max }\right)\right)$. As a result, the t-dependence of the cross section is expressed as the exponential function. Then, V. Yu. Grishina et al. expressed the differential cross section introducing an exponential form factor $F(t)$ :

$$
\begin{aligned}
\frac{d \sigma}{d t} & \sim \frac{g_{1}^{2} g_{2}^{2}}{64 \pi\left|p_{1}\right|^{2} s} F(t)^{2}\left(\frac{s}{s_{0}}\right)^{2 \alpha(t)}, \\
F(t) & =e^{R^{2} t},
\end{aligned}
$$




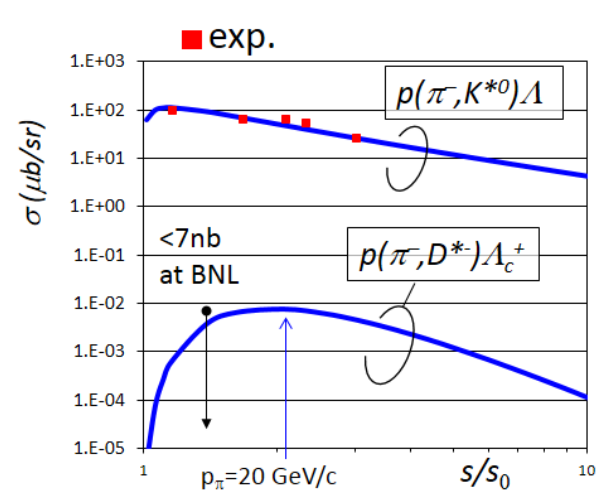

Figure 3: Cross sections calculated based on the Regge model with the Grishina's parameters. The cross sections are normalized so as to reproduce the experimental data of the $p\left(\pi^{-}, K^{*}\right) \Lambda$ reactions [16 17] as plotted with closed squares.

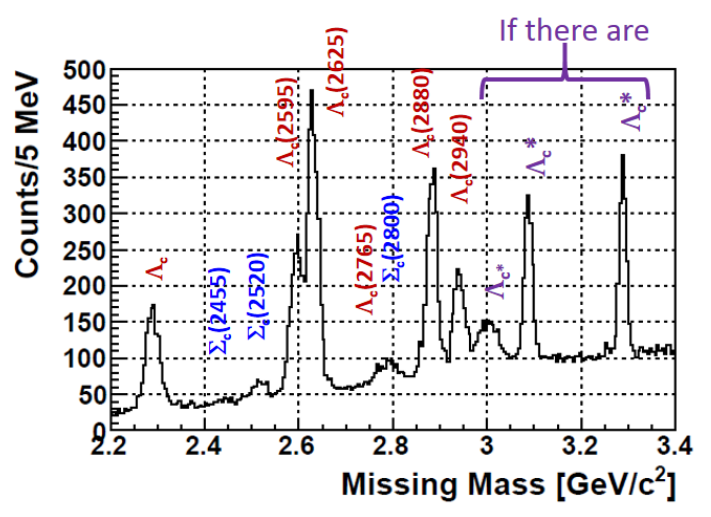

Figure 4: Simulated missing mass spectrum of charmed baryons.

where $R^{2}$ is a parameter related to the so-called slope parameter [15]. Here, $R^{2}=2.13 \mathrm{GeV}^{-1}$ is chosen so as to reproduce the differential cross sections of pion-induced $\Lambda$ hyperon production reactions in a wide range of $s$ [15].

Fig. 3 shows cross sections of the $p\left(\pi^{-}, K^{* 0}\right) \Lambda$ and $p\left(\pi^{-}, D^{*-}\right) \Lambda_{c}^{+}$calculated based on the Regge model with the Grishina's parameters. $K^{*}$ and $D^{*}$ reggeons are assumed respectively. The cross sections are normalized so as to reproduce the experimental data of the $p\left(\pi^{-}, K^{* 0}\right) \Lambda$ reactions [16 17] as plotted with closed squares. The Grishina's model reproduces the $s$-dependence of the data very well. An arrow in the figure indicates the $s / s_{0}$ values for the $p\left(\pi^{-}, D^{*-}\right) \Lambda_{c}^{+}$reaction at the beam momentum of $20 \mathrm{GeV} / c$, where we expect the cross section as large as $10 \mathrm{nb}$.

\subsection{Expected spectrum}

Fig. 4 shows a simulated missing mass spectrum of charmed baryons. In the simulation, the cross sections are assumed to be $1 \mathrm{nb}$ and the estimated production rates shown in Fig. 2f(b) for the ground state $\Lambda_{c}^{+}$and the excited states, respectively. The masses and the decay widths of the charmed baryons reported by the Particle Data Group [18] are used except for three peaks, labeled as " $\Lambda_{c}^{*}$ ", demonstrated as a case if there are. The cross section of $1 \mathrm{nb}$ is assumed for each. A decay width of $\Gamma \sim 100 \mathrm{MeV}$ for the broader peak at around $3 \mathrm{GeV} / \mathrm{c}^{2}$ and no decay widths for the others are taken into account. Background events are generated by using the JAM code [19]. Background ground level is reduced mainly by reconstructing $D^{*-}$ and $\overline{D^{0}}$ masses. A signal to background ratio is further improved by applying some additional cuts, such as selection of a forward region of $D^{*-}$ scattering angles assuming t-channel dominance and discarding forward and backward angles of scattered $K^{+}$or $\pi^{-}$in the rest frame of $\bar{D}^{0}$ [10]. We expect $\sim 1000$ events/nb for 100 days of running time with $10^{7}$ pions per second irradiated on a $4 \mathrm{~g} / \mathrm{cm}^{2}$ hydrogen target. 


\section{Summary and Outlook}

Charmed baryons with a charm quark $\left(Y_{c}\right)$ provide a unique opportunity to investigate quark dynamics, particularly diquark correlations, in baryons. We demonstrated that the nature of light$q q$ diquark motions in charmed baryons could appear in the level structure, production rates, and decay branching ratios. We propose a spectroscopic study of charmed baryons via the $p\left(\pi^{-}, D^{*-}\right)$ reaction at J-PARC. Systematic measurements of the excitation energy spectrum and decays of charmed baryons can be carried out by means of the missing mass technique. Construction of the high-momentum beam line is in progress.

\section{References}

[1] T. Nakano et al., Phys. Rev. C79, 025210(2009).

[2] Note on "Developements in Heavy Quarkonium Spectroscopy", Review of Particle Physics [18].

[3] M. Ida and R. Kobayashi, Prog. Theor. Phys. 36, 846(1966).

[4] M. Anselmino et al., Rev. Mod. Phys. 65, 1199(1993).

[5] R. L. Jaffe, Phys. Rep. 409, 1(2005).

[6] T. Friedmann, Eur. Phys. J. C73 2298(2013).

[7] C. Alexandrou, Ph. de Forcrand, and B. Lucini, Phys. Rev. Lett. 97, 222002(2006).

[8] J. Green, M. Engelhardt, J. Negele, and P. Varilly, AIP Conf. Proc. 1441, 172(2012).

[9] H. Noumi et al., "Charmed Baryon Spectroscopy via the $\left(\pi^{-}, D^{*-}\right)$ reaction", J-PARC P50 Proposal, 2012:

http://www.j-parc.jp/researcher/Hadron/en/Proposal_e.html\#1301

[10] K. Shirotori et al., this volume.

[11] A. Hosaka, private communication, 2013.

[12] J. H. Christensen, et al., Phys. Rev. Lett. 55, 154(1985).

[13] A. B. Kaidalov, Z. Phys. C12, 63(1982).

[14] M. M. Brisudova, L. Burakovsky, and T. Goldman, Phys. Rev. D61, 054013(2000).

[15] V.Yu Grishina, et al., Eur. Phys. J. A25, 141(2005).

[16] O. I. Dahl et al., Phys. Rev. 163 1377(1967).

[17] D. J. Crennell et al., Phys. Rev. D6 1220 (1972).

[18] J. Beringer et al. (Particle Data Group), Phys. Rev. D86, 010001(2012).

[19] Y. Nara: "A Microscopic Transport Code for high energy nuclear collisions", http://quark.phy.bnl.gov/ ynara/ jam/; Y. Nara, et al., Phys. Rev. C61, 024901(2000). 